

\title{
On ultrafriable integers
}

Gérald Tenenbaum

\section{To cite this version:}

Gérald Tenenbaum. On ultrafriable integers. Quarterly Journal of Mathematics, 2015, 66 (1), pp.333351. 10.1093/qmath/hau025 . hal-01281588

\section{HAL Id: hal-01281588 https://hal.science/hal-01281588}

Submitted on 2 Mar 2016

HAL is a multi-disciplinary open access archive for the deposit and dissemination of scientific research documents, whether they are published or not. The documents may come from teaching and research institutions in France or abroad, or from public or private research centers.
L'archive ouverte pluridisciplinaire $\mathbf{H A L}$, est destinée au dépôt et à la diffusion de documents scientifiques de niveau recherche, publiés ou non, émanant des établissements d'enseignement et de recherche français ou étrangers, des laboratoires publics ou privés. 


\title{
On ultrafriable integers
}

\author{
Gérald Tenenbaum
}

\begin{abstract}
Say that an integer $n$ is $y$-ultrafriable if its canonical decomposition is free of prime powers exceeding $y$. We investigate the asymptotic behaviour of the distribution function $\Upsilon(x, y)$, equal to the number of $y$-ultrafriable integers not exceeding $x$. The study being restricted to the range $\psi(y)>2 \log x$ (where $\psi$ denotes Chebyshev's function) by a symmetry argument, and writing $\Psi(x, y)$ for the number of $y$-friable integers not exceeding $x, \beta=\beta(x, y)$ for the saddle-point associated to the Dirichlet series $Z(s, y):=\prod_{p \leqslant y}\left(1-p^{-\left(\nu_{p}+1\right) s}\right) /\left(1-p^{-s}\right)$ where $\nu_{p}:=\lfloor(\log y) / \log p\rfloor$, we obtain full description via a number of effective estimates, qualitative versions of which may be stated
\end{abstract} as follows:

(i) $\Upsilon(x, y) \sim \Psi(x, y) \Leftrightarrow y /(\log x)^{2} \rightarrow \infty \quad(x \rightarrow \infty)$

(ii) $\Upsilon(x, y)=o(\Psi(x, y)) \Leftrightarrow y /(\log x)^{2} \rightarrow 0 \quad(x \rightarrow \infty)$;

(iii) $\Upsilon(x, y) \sim x^{\beta} Z(\beta, y) G\left(\beta \sqrt{\sigma_{2}}\right) \quad\left(x \rightarrow \infty, 2 \log x<\psi(y) \leqslant(\log x)^{3}\right)$.

Here $\sigma_{2}:=\mathrm{d}^{2}\{\log Z(\beta, y)\} / \mathrm{d} s^{2}$ and $G(z):=\mathrm{e}^{z^{2} / 2} \int_{z}^{\infty} \mathrm{e}^{-t^{2} / 2} \mathrm{~d} t / \sqrt{2 \pi}(z>0)$.

Moreover, the phase transition between domains (i) and (ii) is quantitatively described in terms of the Dickman function, the Gaussian behaviour as $\log x$ approaches $\frac{1}{2} \psi(y)$ is made explicit, and a quantitative estimate for the local behaviour with respect to the variable $x$ is derived.

Keywords: Friable integers, integers free of large prime factors, saddle-point method, local behaviour, large deviations in the central limit theorem.

\section{Introduction and statement of results}

A positive integer $n$ is said to be $y$-friable if its largest prime factor $P^{+}(n)$ (with the convention that $P^{+}(1)=1$ ) does not exceed $y$. In the last twenty years, friable integers, and in particular the counting function

$$
\Psi(x, y):=\sum_{\substack{n \leqslant x \\ P^{+}(n) \leqslant y}} 1,
$$

received considerable attention in the literature. In this paper, we investigate a related structure.

Let us say that an integer $n$ is $y$-ultrafriable if no prime power dividing $n$ exceeds $y$. Intrinsically a sieve problem but also relevant to other fields such as irreducibility of polynomials [1], graph theory (see, e.g., [9]), or the study of so-called economical integers $[4],{ }^{(1)}$ the distribution of ultrafriable integers raises interesting methodological questions which hopefully can be fairly satisfactorily answered using available techniques previously developed in the context of friable integers.

Denote by $\Upsilon(x, y)$ the number of $y$-ultrafriable integers not exceeding $x$ and put $\nu_{p}=\nu_{p}(y):=\lfloor(\log y) / \log p\rfloor$ for each prime $p \leqslant y$, so that $p^{\nu_{p}}$ is the largest power of $p$ not exceeding $y$. Writing $N_{y}:=\mathrm{e}^{\psi(y)}$ where $\psi(y):=\sum_{p \leqslant y} \nu_{p} \log p$ is Chebyshev's function, we note that all integers counted in $\Upsilon(x, y)$ are divisors of $N_{y}$, and hence

$$
\Upsilon(x, y)=\tau\left(N_{y}\right)=\prod_{p \leqslant y}\left(1+\nu_{p}\right)=2^{\pi(y)+O(\sqrt{y} / \log y)} \quad\left(x \geqslant N_{y}\right),
$$

2010 Mathematics Subject Classification: primary 11N25; secondary 11N35, 11N37, 11N60.

1. An integer $n$ is said to be economical in base $q$ if its prime factorisation can be written with no more digits that $n$ itself in base $q$. Thus $14=2 \cdot 7,15=3 \cdot 5$ and $16=2^{4}$ are economical in base 10 but $18=2 \cdot 3^{2}$ is not. 
where $\pi(y)$ denotes the number of primes not exceeding $y$.

Next, we observe that

$$
\Upsilon(x, y)=\tau\left(N_{y}\right)-\Upsilon\left(\left(N_{y} / x\right)-, y\right) \quad\left(\sqrt{N_{y}} \leqslant x \leqslant N_{y}\right) .
$$

Therefore, we may restrict the study of $\Upsilon(x, y)$ to the case

$$
x<\sqrt{N_{y}} \text {, i.e. } \psi(y)>2 \log x .
$$

The Dirichlet series associated to the counting function $\Upsilon(x, y)$ is

$$
Z(s, y):=\prod_{p \leqslant y} \frac{1-p^{-\left(\nu_{p}+1\right) s}}{1-p^{-s}} \quad(\Re e s>0) .
$$

While, for large values of $y$, we readily obtain satisfactory estimates for $\Upsilon(x, y)$ from results on the local behaviour of $\Psi(x, y)$ (see [3]), we need to perform a direct evaluation of the Perron integral when $y$ is small. The details are then similar to those appearing in the study of squarefree friable integers provided in [2], with however some significant discrepancies. When it will seem appropriate, we shall skip certain calculations and refer to the corresponding details in [2].

The saddle-point, say $\beta=\beta(x, y)$, relevant to the Perron integral for $\Upsilon(x, y)$ is defined by the equation

$$
\varphi_{1}(\beta, y)=\log x
$$

where

$$
\varphi_{1}(s, y):=\frac{-Z^{\prime}(s, y)}{Z(s, y)}=\sum_{p \leqslant y}\left\{\frac{\log p}{p^{s}-1}-\frac{\left(\nu_{p}+1\right) \log p}{p^{\left(\nu_{p}+1\right) s}-1}\right\} \quad(\Re e s>0, y \geqslant 2) .
$$

Then $\varphi_{1}(\sigma, y)$ is a decreasing function of $\sigma$ such that $\varphi_{1}(0+, y)=\frac{1}{2} \psi(y), \varphi_{1}(\infty, y)=0$. Thus, under assumption (1.2), equation (1.4) has a unique solution and $\beta$ is well defined. For convenience, we put

$$
\beta:=0 \quad(\psi(y) \leqslant 2 \log x) .
$$

We write

$$
\varphi_{j}(s, y):=(-1)^{j-1} \frac{\mathrm{d}^{j-1} \varphi_{1}}{\mathrm{~d} \sigma^{j-1}}(s, y), \quad \sigma_{j}:=\varphi_{j}(\beta, y) \quad(j \geqslant 1),
$$

let

$$
\Phi(z):=\frac{1}{\sqrt{2 \pi}} \int_{z}^{\infty} \mathrm{e}^{-t^{2} / 2} \mathrm{~d} t \quad(z \in \mathbb{R})
$$

denote the decreasing distribution function of the Gaussian law, and put

$$
G(z):=\mathrm{e}^{z^{2} / 2} \Phi(z) \quad(z \in \mathbb{R}) .
$$

Thus

$$
G(z)=\frac{1}{2}+O(z) \quad(z \rightarrow 0), \quad G(z)=\frac{1}{\sqrt{2 \pi} z}\left\{1-\frac{1}{z^{2}}+O\left(\frac{1}{z^{4}}\right)\right\} \quad(z \rightarrow+\infty) .
$$


We also define

$$
\mathcal{H}_{y}(\sigma):=\prod_{p \leqslant y}\left(1-\frac{1}{p^{\left(\nu_{p}+1\right) \sigma}}\right) \quad(\sigma>0),
$$

and make systematic use of the notation

$$
u:=\frac{\log x}{\log y} \quad(x \geqslant y \geqslant 2) .
$$

Theorem 1.1. Let $\varepsilon>0$. For $x \geqslant y \geqslant 2$, we have

$$
\begin{aligned}
& \Upsilon(x, y)=\Psi(x, y)\left\{1+O\left(\frac{u \log 2 u}{\sqrt{y} \log y}\right)\right\} \quad\left(x \geqslant y>(\log x)^{2+\varepsilon}\right) \\
& \Upsilon(x, y)=x^{\beta} Z(\beta, y) G\left(\beta \sqrt{\sigma_{2}}\right)\left\{1+O\left(\frac{1}{u}\right)\right\} \quad\left(2 \log x<\psi(y) \ll(\log x)^{3}\right) .
\end{aligned}
$$

From this result, a number of more explicit estimates may be easily deduced.

Some further notation is necessary to describe the results. Let $\alpha=\alpha(x, y)$ denote the saddle-point of the Perron integral for $\Psi(x, y)$. So $\alpha$ is defined by the equation

$$
\sum_{p \leqslant y} \frac{\log p}{p^{\alpha}-1}=\log x
$$

Explicit approximations to $\alpha$ are widely available in the literature. In particular, it is proved in [5] that

$$
\alpha=\frac{1}{\log y} \log \left(1+\frac{y}{\log x}\right)\left\{1+O\left(\frac{\log _{2} 2 y}{\log y}\right)\right\} \quad(x \geqslant y \geqslant 2),
$$

and, more precisely, that, for any $\varepsilon>0$,

$$
\alpha= \begin{cases}1-\frac{\xi(u)}{\log y}+O\left(\frac{1}{L_{\varepsilon}(y)}+\frac{1}{u(\log y)^{2}}\right) & \left((\log x)^{1+\varepsilon} \leqslant y \leqslant x\right), \\ \frac{\log (1+y / \log x)}{\log y}\left\{1+O\left(\frac{1}{\log y}\right)\right\} & \left(2 \leqslant y \leqslant(\log x)^{3}\right),\end{cases}
$$

where $\xi(v)$ is defined as the unique solution of the equation $\mathrm{e}^{\xi(v)}=1+v \xi(v)$ for $v>1$ and $\xi(1):=0$. Here and in the sequel, we let $\log _{k}$ denote the $k$-fold iterated logarithm and put

$$
L_{\varepsilon}(y):=\mathrm{e}^{(\log y)^{3 / 5-\varepsilon}} \quad(\varepsilon>0, y \geqslant 2) .
$$

Available studies of the local behaviour of the counting function $\Psi(x, y)$ - see in particular th. 2.4 of [3] - provide various effective versions of the approximation

$$
\Psi\left(\frac{x}{h}, y\right) \approx \frac{\Psi(x, y)}{h^{\alpha}} \quad(x \geqslant y \geqslant 2,1 \leqslant h \leqslant x) .
$$

For $P^{+}(d) \leqslant y$, put $h_{d}:=\prod_{p \mid d} p^{\nu_{p}+1}$. Then the characteristic function of $y$-ultrafriable integers may be written as

$$
\sum_{\substack{P^{+}(d) \leqslant y \\ h_{d} \mid n}} \mu(d)
$$


and we have the sieve formula

$$
\Upsilon(x, y)=\sum_{P^{+}(d) \leqslant y} \mu(d) \Psi\left(\frac{x}{h_{d}}, y\right) \quad(x \geqslant y \geqslant 2) .
$$

Considering (1.16), this leads to the expectation that, in suitable ranges, we should have

$$
\Upsilon(x, y) \sim \Psi(x, y) \sum_{P^{+}(d) \leqslant y} \frac{\mu(d)}{h_{d}^{\alpha}}=\Psi(x, y) \mathcal{H}_{y}(\alpha) .
$$

Our first corollary below shows that the right-hand side of (1.17) always constitutes an upper bound for the order of magnitude of $\Upsilon(x, y)$ and that this turns into a lower bound when $\alpha$ is replaced by $\beta$. The second part of the statement provides the exact domain of validity of $(1 \cdot 17)$.

We introduce the remainder term

$$
E=E(x, y):= \begin{cases}\frac{u \log 2 u}{\sqrt{y}} & \text { if } y>\frac{(\log x)^{3}}{\log _{2} 2 x}, \\ \frac{1}{\sqrt{u}}+\frac{(\log x)^{3}}{y^{2} \log _{2} 2 x} & \text { if } 2 \leqslant y \leqslant \frac{(\log x)^{3}}{\log _{2} 2 x}\end{cases}
$$

and note that

$$
E \ll \sqrt{\frac{\log _{2} 2 x}{\log x}}+\frac{(\log x)^{3}}{y^{2} \log _{2} 2 x} \quad(x \geqslant y \geqslant 2) .
$$

Corollary 1.2. (i) We have

$$
\mathcal{H}_{y}(\beta) \Psi(x, y) \ll \Upsilon(x, y) \ll \mathcal{H}_{y}(\alpha) \Psi(x, y) \quad(x \geqslant y \geqslant 2) .
$$

(ii) As $x \rightarrow \infty$, we have

$$
\begin{aligned}
\Upsilon(x, y) & \sim \mathcal{H}_{y}(\alpha) \Psi(x, y) \Leftrightarrow y \sqrt{\log _{2} x} /(\log x)^{3 / 2} \rightarrow \infty \\
\Upsilon(x, y) & \sim \mathcal{H}_{y}(\beta) \Psi(x, y) \Leftrightarrow y \sqrt{\log _{2} x} /(\log x)^{3 / 2} \rightarrow \infty
\end{aligned}
$$

Moreover,

$$
\begin{array}{ll}
\Upsilon(x, y)=\{1+O(E)\} \mathcal{H}_{y}(\alpha) \Psi(x, y) & \left(y \gg(\log x)^{3 / 2} / \sqrt{\log _{2} x}\right) \\
\Upsilon(x, y)=\{1+O(E)\} \mathcal{H}_{y}(\beta) \Psi(x, y) & \left(y \gg(\log x)^{3 / 2} / \sqrt{\log _{2} x}\right)
\end{array}
$$

In view of $(1 \cdot 6)$, the lower bound in $(1 \cdot 18)$ vanishes when $\psi(y) \leqslant 2 \log x$. However, as observed earlier, we have $\frac{1}{2} \tau\left(N_{y}\right) \leqslant \Upsilon(x, y) \leqslant \tau\left(N_{y}\right)$ in this circumstance.

As is already apparent in the statement of Theorem 1.1, the asymptotic fluctuations of $\Upsilon(x, y)$ present a threshold around $y \approx(\log x)^{2}$. Our next corollary exhibits the behaviours on either side of this threshold and describes the phase transition.

We recall that the Dickman function $\varrho: \mathbb{R}^{+} \rightarrow[0,1]$ is defined as the continuous solution to the delay differential equation $v \varrho^{\prime}(v)+\varrho(v-1)=0$ such that $\varrho(v)=1$ for $0 \leqslant v \leqslant 1$. An explicit expression of its Laplace transform is well-known (see, e.g., [11], th. III.5.10):

$$
\widehat{\varrho}(s)=\int_{0}^{\infty} \mathrm{e}^{-s v} \varrho(v) \mathrm{d} v=\mathrm{e}^{\gamma+I(-s)}, \quad I(s):=\int_{0}^{s} \frac{\mathrm{e}^{t}-1}{t} \mathrm{~d} t,
$$


where $\gamma$ denotes Euler's constant. ${ }^{(2)}$ On the real axis, $\varrho$ has a simple behaviour:

$$
\widehat{\varrho}(t)=\frac{1+O\left(\mathrm{e}^{-t} / t\right)}{t} \quad(t \rightarrow+\infty), \quad \widehat{\varrho}(t)=\exp \left\{\frac{\mathrm{e}^{|t|}}{|t|}+O\left(\frac{\mathrm{e}^{|t|}}{|t|^{2}}\right)\right\} \quad(t \rightarrow-\infty) .
$$

Corollary 1.3. As $x \rightarrow \infty$, we have

$$
\begin{gathered}
\Upsilon(x, y) \sim \Psi(x, y) \Leftrightarrow y /(\log x)^{2} \rightarrow \infty, \\
\Upsilon(x, y)=o(\Psi(x, y)) \Leftrightarrow y /(\log x)^{2} \rightarrow 0 .
\end{gathered}
$$

Moreover, for any $\varepsilon>0$, and uniformly for $(\log x)^{2} / L_{\varepsilon}(\log x) \leqslant y \leqslant(\log x)^{5 / 2} /\left(\log _{2} x\right)^{3 / 2}$, we have

$$
\Upsilon(x, y)=\left\{1+O\left(\frac{1}{\sqrt{u}}\right)\right\} \mathcal{H}_{y}(\beta) \Psi(x, y)=\left\{1+O\left(\frac{1}{L_{\varepsilon}(y)}\right)\right\} \frac{\widehat{\varrho}(h)}{2 \widehat{\varrho}(2 h)} \Psi(x, y)
$$

with $h:=\frac{1}{2} \log y-\xi(u)$.

To clarify expectations, we note that the parameter $h$ appearing in (1.26) satisfies $\mathrm{e}^{h} \sim 2 \sqrt{y} / \log x$ in the critical range $y=(\log x)^{2+o(1)}$. We also observe that when $y>(\log x)^{5 / 2} /\left(\log _{2} x\right)^{3 / 2}$, the estimate $(1 \cdot 10)$ is more precise than $(1 \cdot 26)$.

The following result concerns smaller values of $y$, when the saddle-point estimates for $\Upsilon(x, y)$ and $\Psi(x, y)$ assume different shapes. This corresponds essentially to the case $2 \log x<\psi(y)<(2+c) \log x$ for suitable $c \in] 0, \frac{1}{2}[$. We obtain a large deviation result which measures the Gaussian distribution of the divisors of $N_{y}=\mathrm{e}^{\psi(y)}$. The proof will be omitted since it is identical, mutatis mutandis, to that of Corollary 2.2 of [2].

Corollary 1.4. Let $y \geqslant 2, N_{y}=\mathrm{e}^{\psi(y)}, D_{y}^{2}:=\frac{1}{12} \sum_{p \leqslant y} \nu_{p}\left(\nu_{p}+2\right)(\log p)^{2}$. Uniformly for $0 \leqslant z \ll(y / \log y)^{1 / 4}$ and $x=\sqrt{N_{y}} \mathrm{e}^{-z D_{y}}$, we have

$$
\Upsilon(x, y)=\tau\left(N_{y}\right) \Phi(z)\left\{1+O\left(\frac{1+z^{4}}{u}\right)\right\} .
$$

According to a remark developed in [2] and still valid in the present context, we note that Petrov's effective theorem on large deviations in the central limit theorem (see [7], th. VIII.2) provides estimates that are similar in nature to, but less precise than (1.27) in its range of validity.

Finally, as a specific by-product of saddle-point asymptotic formulae, we state a result on the local behaviour of $\Upsilon(x, y)$. We also omit the proof, since it is identical to that of corollary 2.4 of [3], ${ }^{(3)}$ and furthermore leave to the reader the possibility of deriving corresponding short interval estimates parallel to theorem 2.5 and corollary 2.6 of [2].

Corollary 1.5. Uniformly under the conditions $x \geqslant y \geqslant 2,1 \leqslant d \leqslant y, \psi(y)>2 \log (d x)$, we have

$$
\Upsilon(d x, y)=d^{\beta} \Upsilon(x, y)\left\{1+O\left(\frac{1}{\sqrt{u}}\right)\right\}
$$

2. We shall take the liberty to use the letter $\gamma$ for other purposes later in the paper.

3. Apart from the simplification due to the fact that, in view of (1.10), the required result follows directly, for $y>(\log x)^{3}$, from known results on the local behaviour of $\Psi(x, y)$ - see [3]. 


\section{Lemmas}

We start with a useful elementary inequality.

Lemma 2.1. Let $\nu \in \mathbb{N}^{*}, z>1$. Then

$$
\frac{1}{z+1} \leqslant \frac{1}{z-1}-\frac{\nu+1}{z^{\nu+1}-1} \leqslant \frac{\nu}{z+1} .
$$

Moreover, the right-hand inequality is strict when $\nu \geqslant 2$.

Proof. The left-hand inequality is equivalent to

$$
\frac{\nu+1}{z^{\nu+1}-1} \leqslant \frac{1}{z-1}-\frac{1}{z+1}=\frac{2}{z^{2}-1} .
$$

This is clear since $\left(z^{v}-1\right) / v$ is an increasing function of $v>0$ when $z>1$.

To prove the right-hand inequality, we may assume $\nu \geqslant 2$ since we trivially have equality when $\nu=1$. Then, the required inequality may be rewritten as

$$
(z+1)\left(z^{\nu+1}-1\right)<(\nu+1)\left(z^{2}-1\right)+\nu(z-1)\left(z^{\nu+1}-1\right),
$$

which, after straightforward transformations, amounts to

$$
f(z):=(\nu-1)\left(z^{\nu}+1\right)-2 \sum_{1 \leqslant j<\nu} z^{j}>0 .
$$

However, we have $f(1)=0$, and $f^{\prime}(z)=\nu(\nu-1) z^{\nu-1}-2 \sum_{1 \leqslant j<\nu} j z^{j-1}>0$ for $z>1$.

Our next lemma provides uniform estimates for the sums

$$
g_{ \pm}(\sigma, y):=\sum_{p \leqslant y} \frac{\log p}{p^{\sigma} \pm 1}
$$

as observed in [2], these may be proved by partial summation from a strong form of the prime number theorem along the lines described in [5], lemma 13. We omit the details.

Lemma 2.2. Uniformly for $0<\sigma<2, y \geqslant 2$, we have

$$
g_{ \pm}(\sigma, y)=\frac{y^{1-\sigma}-1}{\left(1 \pm y^{-\sigma}\right)(1-\sigma)}\left\{1+O\left(\frac{1}{\log y}\right)\right\}+O(1)
$$

Moreover, given any $\sigma_{0}>0, \varepsilon>0$, the remainder term $O(1 / \log y)$ may be replaced by $O\left(1 / L_{\varepsilon}(y)\right)$ when $\sigma \geqslant \sigma_{0}$.

We shall also need an estimate for the order of magnitude of the quantity

$$
V_{y}(\sigma):=\sum_{p \leqslant y} \frac{\left(\nu_{p}+1\right) \log p}{p^{\left(\nu_{p}+1\right) \sigma}-1} \quad(\sigma>0) .
$$

Lemma 2.3. For $y \geqslant 2,1 / \log y<\sigma \leqslant 2$, we have

$$
V_{y}(\sigma) \asymp \frac{y^{1 / 2-\sigma}\left(1+y^{1 / 2-\sigma}\right) \log y}{1+|1-2 \sigma| \log y} .
$$

Proof. We have $V_{y}(\sigma) \asymp(S+T) \log y$, with

$$
S:=\sum_{p \leqslant \sqrt{y}} \frac{1}{p^{\left(\nu_{p}+1\right) \sigma}}, \quad T:=\sum_{\sqrt{y}<p \leqslant y} \frac{1}{p^{2 \sigma}} .
$$


By the inequality $p^{\nu_{p}+1}>y$ and the prime number theorem, we may write

$$
S \ll \frac{y^{1 / 2-\sigma}}{\log y}, \quad T \asymp \frac{y^{1-2 \sigma}-y^{1 / 2-\sigma}}{(1-2 \sigma) \log y} \asymp \frac{y^{1 / 2-\sigma}\left(1+y^{1 / 2-\sigma}\right)}{1+|1-2 \sigma| \log y},
$$

in view of the uniform estimate

$$
\frac{\mathrm{e}^{t}-1}{t} \asymp \frac{1+\mathrm{e}^{t}}{1+|t|} \quad(t \in \mathbb{R}) .
$$

Thus $S \ll T$, whence $V_{y}(\sigma) \asymp T \log y$, as required.

The following result provides explicit estimates for $\beta$ in terms of $x$ and $y$. The asymptotic behaviour of the function $\xi$ appearing in (1.14) has been described in [6]. In particular, we have

$$
\xi(v)=\log v+\log _{2} v+\frac{\log _{2} v}{\log v}+O\left(\left(\frac{\log _{2} v}{\log v}\right)^{2}\right) \quad(v \geqslant 3) .
$$

Lemma 2.4. Let $\varepsilon>0$.

(i) For $x \geqslant x_{0}(\varepsilon),(\log x)^{1+\varepsilon}<y \leqslant x$, we have

$$
\beta=1-\frac{\xi(u)}{\log y}+O\left(\frac{1}{u(\log y)^{2}}+\frac{1}{L_{\varepsilon}(y)}\right) .
$$

(ii) For $x \geqslant 2,2 \log x<\psi(y) \ll(\log x)^{3}$, we have

$$
\beta=\frac{1+O(1 / \log y)}{\log y} \log \left(\frac{\psi(y)}{\log x}-1\right) .
$$

(iii) For $x \geqslant y \geqslant 2, \psi(y)>2 \log x, r:=y /(\log x)^{2}$, we have,

$$
\alpha-\beta \asymp \frac{(\log 2 u)(\sqrt{y}+u \log 2 u)}{y(\log y)(1+|\log r|)} .
$$

Proof. We note that, in its range of validity, $(2 \cdot 9)$ follows from $(2 \cdot 11)$ in view of $(1 \cdot 14)$. However, it will be convenient to derive $(2 \cdot 9)$ as a preliminary step. By $(2 \cdot 1)$, we have, for all $\sigma>0$,

$$
g_{+}(\sigma, y) \leqslant \varphi_{1}(\sigma, y) \leqslant g_{+}(\sigma, y)+r(\sigma, y),
$$

say, with

$$
r(\sigma, y):=\sum_{p \leqslant \sqrt{y}} \frac{\left(\nu_{p}-1\right) \log p}{p^{\sigma}+1}
$$

Let us first assume $y>(\log x)^{3}$. Then we deduce from (2.4) and the left-hand inequality above that $\beta>3 / 5$ provided $x$ is sufficiently large. Inserting this back into (1.5) and taking $(2 \cdot 6)$ into account, we obtain

$$
\varphi_{1}(\beta, y)=g_{-}(\beta, y)+O\left(y^{-1 / 10}\right) .
$$


This is sufficient to deduce $(2 \cdot 9)$ by computations identical to those leading to estimate (7.8) of [5].

Next, we consider the case $(\log x)^{1+\varepsilon}<y \leqslant(\log x)^{3}$. Then it follows from $(2 \cdot 8)$ and $(2 \cdot 9)$ that $\beta \leqslant \beta\left(x, 2(\log x)^{3}\right) \leqslant \frac{2}{3}+O(1 / \log y)$. Therefore

$$
r(\beta, y) \ll(\log y) \sum_{p \leqslant \sqrt{y}} \frac{1}{p^{\beta}} \ll y^{(1-\beta) / 2}
$$

where the last bound readily follows by partial summation - see lemma 3.6 of [3] for a general estimate. We hence deduce from $(2 \cdot 4)$ that

$$
r(\beta, y) \ll g_{+}(\beta, y) y^{-1 / 6} .
$$

Thus we obtain that the estimate $(2 \cdot 4)$ for $g_{+}(\beta, y)$ equally holds for $\varphi_{1}(\beta, y)$. We may now again deduce $(2 \cdot 9)$ by computations parallel to those leading to estimate (7.8) of [5]. We refer the reader to [5] for the details.

Let us next evaluate $\beta$ when $2 \log x<\psi(y) \leqslant(\log x)^{3}$. In this range, it follows from $(2 \cdot 14)$ that

$$
g_{+}(\beta, y)=\left\{1+O\left(\frac{1}{y^{1 / 6}}\right)\right\} \log x
$$

By $(2 \cdot 4)$, and since we have $\beta<3 / 4$, this plainly implies

$$
y^{1-\beta} \asymp \log x .
$$

Now, by $(2 \cdot 1)$ with $z=p^{\beta}$ and $\nu=\nu_{p}=\nu_{p}(y)$,

$$
-\sum_{p \leqslant \sqrt{y}} \frac{\left(\nu_{p}-1\right) \log p}{1+p^{\beta}} \leqslant \varphi_{1}(\beta, y)-\frac{\psi(y)}{1+y^{\beta}} \leqslant \sum_{p \leqslant y} \frac{\nu_{p}(y)\left(y^{\beta}-p^{\beta}\right) \log p}{\left(1+p^{\beta}\right)\left(1+y^{\beta}\right)} .
$$

By partial summation, we obtain that the above upper bound is $\ll \beta y^{1-\beta} \ll \beta \log x$. Moreover, by $(2 \cdot 14)$ and $(2 \cdot 15)$, the lower bound is

$$
-r(\beta, y) \ll \frac{\log x}{y^{1 / 6}} .
$$

Let $\gamma$ denote the solution to the equation $\psi(y) /\left(1+y^{\gamma}\right)=\log x$. We deduce from the above that

$$
|\beta-\gamma|(\log x) \log y \ll \frac{\psi(y)\left|y^{\beta}-y^{\gamma}\right|}{\left(1+y^{\beta}\right)\left(1+y^{\gamma}\right)} \ll\left(\beta+\frac{1}{y^{1 / 6}}\right) \log x
$$

This yields $(2 \cdot 10)$ if, say, $\psi(y)>\left(2+y^{-1 / 7}\right) \log x$. In the complementary case, we appeal to the estimate

$$
\varphi_{1}(\sigma, y)=\frac{1}{2} \psi(y)-\sigma D_{y}^{2}+O\left(\sigma^{2} y(\log y)^{2}\right) \quad\left(0 \leqslant \sigma \log y<\frac{1}{2}\right)
$$

where $D_{y}^{2}$ is defined in Corollary 1.4 and satisfies $D_{y}^{2}=\frac{1}{4} y \log y+O(y)$. This readily follows from the classical expansion of $z /\left\{\mathrm{e}^{z}-1\right\}$ involving Bernoulli numbers, up to the third order. Substituting $\sigma=\beta$ yields $(2 \cdot 10)$. 
We are now in a position to prove the more precise estimate $(2 \cdot 11)$. From $(1 \cdot 14),(2 \cdot 9)$ and $(2 \cdot 10)$, we see that, for any $\vartheta \in[0,1]$ and $\gamma=\alpha+\vartheta(\beta-\alpha)$, we have in the considered range

$$
g_{-}^{\prime}(\gamma, y)=\mathrm{e}^{O(R)} \frac{\left(y^{1-\gamma}-1\right) \log y}{\left(1-y^{-\gamma}\right)^{2}(1-\gamma)} \asymp u(\log y)^{2},
$$

with $R:=1 / \log 2 u+1 / \log y$, where the first estimate is proved in lemmas 4 and 13 of [5], using a strong form of the prime number theorem. Moreover, recalling the notations $(2 \cdot 5)$ and $r:=y /(\log x)^{2}$, we have

$$
\begin{aligned}
g_{-}(\beta, y)-g_{-}(\alpha, y) & =g_{-}(\beta, y)-\varphi_{1}(\beta, y)=\sum_{p \leqslant y} \frac{\left(\nu_{p}+1\right) \log p}{p^{\left(\nu_{p}+1\right) \beta}-1} \\
& =V_{y}(\beta) \asymp \frac{u(\log 2 u)(\sqrt{y}+u \log 2 u) \log y}{y(1+|\log r|)},
\end{aligned}
$$

where the last estimate follows from $(2 \cdot 6),(2 \cdot 9)$ and $(2 \cdot 10)$, since

$$
y^{1 / 2-\beta} \asymp \frac{u \log 2 u}{\sqrt{y}} \quad(\psi(y)>2 \log x) .
$$

Estimate $(2 \cdot 11)$ readily follows from this and $(2 \cdot 17)$, by the mean-value theorem.

We next evaluate the derivatives $\sigma_{j}(j \geqslant 1)$ defined in $(1 \cdot 7)$ for comparatively small values of $y$.

Lemma 2.5. Let $j \in \mathbb{N}^{*}$ be fixed. Uniformly for $2 \log x<\psi(y) \ll(\log x)^{3}$, we have

$$
\sigma_{j} \ll u(\log y)^{j} .
$$

For $j=3$, the right-hand side may be multiplied by $\min (1, \beta \log y)+1 / \sqrt{u}$. Moreover, when $j=2$, we may replace the «-sign by $\asymp$. More precisely,

$$
\sigma_{2}=\left\{1+O\left(\frac{1}{\log y}\right)\right\} \frac{w-1}{w} u(\log y)^{2}
$$

with $w:=\psi(y) / \log x$.

Proof. Put $R_{p}(z):=\sum_{0 \leqslant h \leqslant \nu_{p}} z^{h}$. A simple induction provides the formula

$$
\sigma_{j}=\sum_{p \leqslant y} \frac{Q_{j, p}\left(p^{\beta}\right)(\log p)^{j}}{R_{p}\left(p^{\beta}\right)^{j}}
$$

where $Q_{j, p}$ is a polynomial of degree $\nu_{p} j-1$ with coefficients $\ll \nu_{p}^{j}$. This immediately implies $(2 \cdot 20)$ in view of the first inequality in $(2 \cdot 12)$.

Recall that our hypotheses imply $\beta \leqslant \frac{2}{3}+O(1 / \log y)$. To prove the complementary assertions, we observe that, for any fixed $j \geqslant 1$,

$$
\begin{aligned}
\sigma_{j}-\frac{\mathrm{d}^{j-1} g_{+}}{\mathrm{d} \sigma^{j-1}}(\beta, y) & =-\frac{\mathrm{d}^{j-1} g_{+}}{\mathrm{d} \sigma^{j-1}}(\beta, \sqrt{y})+\sum_{p \leqslant \sqrt{y}} \frac{Q_{j, p}\left(p^{\beta}\right)(\log p)^{j}}{R_{p}\left(p^{\beta}\right)^{j}} \\
& \ll(\log y)^{j} \sum_{p \leqslant \sqrt{y}} \frac{1}{p^{\beta}} \ll(\log y)^{j-1} y^{(1-\beta) / 2} \ll \sqrt{u}(\log y)^{j} .
\end{aligned}
$$

Since

$$
\frac{\mathrm{d}^{2} g_{+}}{\mathrm{d} \sigma^{2}}(\beta, y)=\sum_{p \leqslant y} \frac{(\log p)^{3} p^{\beta}\left(p^{\beta}-1\right)}{\left(1+p^{\beta}\right)^{3}} \ll \min (1, \beta \log y) u(\log y)^{3},
$$

we obtain the statement regarding the case $j=3$. 
As for the case $j=2$, we first note, on applying the prime number theorem as for the proof of $(2 \cdot 4)$, that the estimate

$$
g_{+}^{\prime}(\sigma, y)=\left\{1+O\left(\frac{1}{\log y}\right)\right\} \frac{1}{\left(1+y^{-\sigma}\right)^{2}} \int_{1}^{y} \frac{\log t}{t^{\sigma}} \mathrm{d} t
$$

holds uniformly for $\sigma>0$. Taking $(2 \cdot 4)$ and $(2 \cdot 15)$ into account and evaluating $1+y^{-\beta}$ by $(2 \cdot 10)$, we get

$$
g_{+}^{\prime}(\beta, y)=\mathrm{e}^{O(R)} \frac{w-1}{w} u(\log y)^{2},
$$

with $R:=1 / \log 2 u+1 / \log y \ll 1 / \log y$. By $(2 \cdot 22)$, this estimate is equally valid for $\sigma_{2}$ : indeed $\sqrt{u}>y^{1 / 7}$ in the range under study.

We shall need the following estimate to control the decay of $|Z(s, y)|$ along the line $\sigma=\beta$. We write $s=\beta+i \tau$ with $\tau \in \mathbb{R}$ and set

$$
Y_{\varepsilon}:=\mathrm{e}^{(\log y)^{3 / 2-\varepsilon}} \quad(y \geqslant 2) .
$$

Lemma 2.6. Let $\varepsilon>0$. For a suitable absolute constant $c>0$, we have

$$
\left|\frac{Z(\beta+i \tau, y)}{Z(\beta, y)}\right| \leqslant \begin{cases}\mathrm{e}^{-c u(\tau \log y)^{4}} & \text { if }|\tau| \leqslant 1 / \log y, \\ \mathrm{e}^{-c u \tau^{4} /\left(1+\tau^{4}\right)} & \text { if } 1 / \log y<|\tau| \leqslant Y_{\varepsilon} .\end{cases}
$$

Proof. For $s=\beta+i \tau(\tau \in \mathbb{R})$, a standard computation yields

$$
\left|\frac{Z(s, y)}{Z(\beta, y)}\right|^{2}=\prod_{p \leqslant y} \frac{1+4 \sin ^{2}\left(\frac{1}{2} \tau\left(\nu_{p}+1\right) \log p\right) /\left\{p^{\beta\left(\nu_{p}+1\right)}\left(1-p^{-\beta\left(\nu_{p}+1\right)}\right)^{2}\right\}}{1+4 \sin ^{2}\left(\frac{1}{2} \tau \log p\right) /\left\{p^{\beta}\left(1-p^{-\beta}\right)^{2}\right\}} .
$$

Now, observe that

$$
\nu^{2} \leqslant\left(\sum_{0 \leqslant j<\nu} p^{j \beta}\right)\left(\sum_{0 \leqslant j<\nu} p^{-j \beta}\right)=p^{(\nu-1) \beta}\left(\frac{1-p^{-\nu \beta}}{1-p^{-\beta}}\right)^{2} \quad(\nu \geqslant 1)
$$

and, by lemma 1 of [10],

$$
\left|\frac{\sin \nu \vartheta}{\nu \sin \vartheta}\right| \leqslant 1-\frac{2}{3} \min \left(1, \nu^{2}\|\vartheta / \pi\|^{2}\right) \leqslant 1-\frac{2}{3}\|\vartheta / \pi\|^{2} \quad(\vartheta \in \mathbb{R}, \nu \geqslant 1)
$$

where $\|z\|$ denotes the distance from the real number $z$ to the set of integers. Therefore, writing $\vartheta_{p}:=\|(\tau / 2 \pi) \log p\|$ and $B_{p}:=p^{\beta}\left(1-p^{-\beta}\right)^{2}$, we obtain that the generic factor in $(2 \cdot 25)$ does not exceed

$$
\begin{gathered}
\frac{1+4 \sin ^{2}\left(\frac{1}{2} \tau\left(\nu_{p}+1\right) \log p\right) /\left\{\left(\nu_{p}+1\right)^{2} B_{p}\right\}}{1+4 \sin ^{2}\left(\pi \vartheta_{p}\right) / B_{p}} \leqslant \frac{B_{p}+4\left(1-2 \vartheta_{p}^{2} / 3\right)^{2} \sin ^{2}\left(\pi \vartheta_{p}\right)}{B_{p}+4 \sin ^{2}\left(\pi \vartheta_{p}\right)} \\
\leqslant \frac{B_{p}+4\left(1-2 \vartheta_{p}^{2} / 3\right) \sin ^{2}\left(\pi \vartheta_{p}\right)}{B_{p}+4 \sin ^{2}\left(\pi \vartheta_{p}\right)}=1-\frac{8 \vartheta_{p}^{2} \sin ^{2}\left(\pi \vartheta_{p}\right)}{3 B_{p}+12 \sin ^{2}\left(\pi \vartheta_{p}\right)},
\end{gathered}
$$

and so, appealing to the lower bound $\left|\sin \left(\pi \vartheta_{p}\right)\right| \geqslant 2 \vartheta_{p}$, we arrive at

$$
\left|\frac{Z(s, y)}{Z(\beta, y)}\right|^{2} \leqslant \mathrm{e}^{-W}
$$


say, with

$$
W:=\frac{8}{3} \sum_{p \leqslant y} \frac{4 \vartheta_{p}^{4}}{16 \vartheta_{p}^{2}+p^{\beta}\left(1-p^{-\beta}\right)^{2}} \gg \sum_{p \leqslant y} \frac{\|(\tau / 2 \pi) \log p\|^{4}}{p^{\beta}} .
$$

If $|\tau| \leqslant 1 / \log y$, we have

$$
W \gg \tau^{4} \sum_{p \leqslant y} \frac{(\log p)^{4}}{p^{\beta}} \gg \frac{\tau^{4}(\log y)^{3}\left(y^{1-\beta}-1\right)}{1-\beta} \gg(\tau \log y)^{4} u
$$

where, for the last estimate, we used $(2 \cdot 9)$ if $y>(\log x)^{3}$ and $(2 \cdot 16)$ in the complementary case.

If $1 / \log y<|\tau| \leqslant \sqrt{y}$, we appeal to upper bounds on primes in short intervals and argue as in lemma 5.12 of [8] to show that

$$
\sum_{z / 2<p \leqslant z}\|(\tau / 2 \pi) \log p\|^{4} \gg \frac{\tau^{4}}{1+\tau^{4}} \frac{z}{\log z} \quad\left(y^{3 / 4}<z \leqslant y\right),
$$

from which we infer by partial summation that $W \gg \tau^{4} u /\left(1+\tau^{4}\right)$, as required.

When $\sqrt{y}<|\tau| \leqslant Y_{\varepsilon}$, we note that the left-hand side of $(2 \cdot 28)$ is

$$
\gg \sum_{z / 2<p \leqslant z} \sin ^{4}\left(\frac{1}{2} \tau \log p\right)=\frac{3}{8} \sum_{z / 2<p \leqslant z} 1-\frac{1}{2} \sum_{z / 2<p \leqslant z}\left\{\cos (\tau \log p)-\frac{1}{4} \cos (2 \tau \log p)\right\} .
$$

However, classical bounds on exponential sums over primes ${ }^{(4)}$ yield that the last sum is $o(z / \log z)$ for $y^{3 / 4}<z \leqslant y$. We may then conclude by partial summation as before.

By a standard procedure, we deduce from the previous lemma an upper estimate for the number of ultrafriable integers in short intervals.

Lemma 2.7. Let $\varepsilon>0$. For a suitable absolute constant $c_{0}>0$, and uniformly for $x \geqslant y \geqslant 2,1 \leqslant z \leqslant \min \left(Y_{\varepsilon}, \mathrm{e}^{c_{0} u}\right)$, we have

$$
\Upsilon(x+x / z, y)-\Upsilon(x, y) \ll x^{\beta} Z(\beta, y) / z .
$$

Proof. From $(2 \cdot 24)$ and a classical bound for short sums of coefficients of Dirichlet series (see e.g. [11], Exercise 171) we infer that the left-hand side of $(2 \cdot 29)$ is

$$
\begin{aligned}
& \ll \frac{x^{\beta}}{z} \int_{0}^{z}|Z(\beta+i \tau, y)| \mathrm{d} \tau \\
& \ll \frac{x^{\beta} Z(\beta, y)}{z}\left\{\int_{0}^{1 / \log y} \mathrm{e}^{-c u(\tau \log y)^{4}} \mathrm{~d} \tau+\int_{1 / \log y}^{z} \mathrm{e}^{-c \tau^{4} u /\left(1+\tau^{4}\right)} \mathrm{d} \tau\right\} \\
& \ll \frac{x^{\beta} Z(\beta, y)}{z}\left\{\frac{1}{u^{1 / 4}}+z \mathrm{e}^{-c u / 2}\right\} .
\end{aligned}
$$

4. See, e.g., [11], equation (III.5.72). 


\section{Proofs}

\subsection{Proof of Theorem 1.1}

Recall the definition (1.12) for the saddle-point $\alpha=\alpha(x, y)$ related to the distribution of friable integers.

First, let us assume $y>(\log x)^{2+\varepsilon}$ with, say, $\left.\varepsilon \in\right] 0, \frac{1}{3}[$. Then, for large $x$, we have $\alpha>\frac{1}{2}+\frac{1}{5} \varepsilon$ by (1.13). By theorem 2.4(i) of [3] the number of integers $n \leqslant x$ which are $y$-friable but not $y$-ultrafriable does not exceed

$$
\sum_{p \leqslant y} \Psi\left(\frac{x}{p^{\nu_{p}+1}}, y\right) \ll \Psi(x, y) S
$$

with

$$
S:=\sum_{p \leqslant y} \frac{1}{p^{\left(\nu_{p}+1\right) \alpha}} \ll \frac{y^{1 / 2-\alpha}}{\log y} \ll \frac{u \log 2 u}{\sqrt{y} \log y}
$$

where the first estimate follows from $(2 \cdot 6)$ and the last from $(1 \cdot 14)$. This proves $(1 \cdot 10)$.

Thus, it remains to prove the estimate (1.11) when

$$
2 \log x<\psi(y) \ll(\log x)^{3} .
$$

In this range, we apply the saddle-point method in a very similar fashion to that of [5]. For purposes of convenience, we note at the outset that the expected main term has order of magnitude

$$
\frac{x^{\beta} Z(\beta, y)}{1+\beta \sqrt{\sigma_{2}}} \gg \frac{x^{\beta} Z(\beta, y)}{\sqrt{u} \log u}
$$

This follows from $(1 \cdot 9),(2 \cdot 10)$ and $(2 \cdot 21)$.

First, we apply Perron's formula with remainder (see [11], th. II.2.3) to get

$$
\Upsilon(x, y)=\frac{1}{2 \pi i} \int_{\beta-i T}^{\beta+i T} \frac{x^{s} Z(s, y)}{s} \mathrm{~d} s+O\left(\frac{x^{\beta} Z(\beta, y) \log T}{T}\right)
$$

with $T:=\mathrm{e}^{2 c_{1}(\log u)^{4 / 3}}<\min \left(\mathrm{e}^{c_{0} u}, Y_{1 / 20}\right)$ where $c_{1}$ is absolute, sufficiently small, and $c_{0}$ is the constant appearing in the statement of Lemma 2.7. This is proved in a standard way using $(2 \cdot 29)$ and we omit the details.

Set $T_{0}:=u^{-1 / 5} / \log y$. The contribution of the range $T_{0} \leqslant|\tau| \leqslant T$ to the last integral may be bounded above using $(2 \cdot 24)$. We obtain that it is

$$
\begin{aligned}
& \ll x^{\beta} Z(\beta, y)\left\{\int_{T_{0}}^{1 / \log y} \mathrm{e}^{-c u(\tau \log y)^{4}} \frac{\mathrm{d} \tau}{\beta+\tau}+\int_{1 / \log y}^{T} \mathrm{e}^{-c u \tau^{4} /\left(1+\tau^{4}\right)} \frac{\mathrm{d} \tau}{\tau}\right. \\
& \ll x^{\beta} Z(\beta, y)\left\{\mathrm{e}^{-c u\left(T_{0} \log y\right)^{4}} \log u+\mathrm{e}^{-\frac{1}{2} c u /(\log y)^{4}} \log _{2} y+\mathrm{e}^{-u / 2} \log T\right\} \\
& \ll x^{\beta} Z(\beta, y) \mathrm{e}^{-c_{2} u^{1 / 5}} .
\end{aligned}
$$

Therefore, under assumption $(3 \cdot 2)$, we get

$$
\Upsilon(x, y)=\frac{1}{2 \pi i} \int_{\beta-i T_{0}}^{\beta+i T_{0}} \frac{x^{s} Z(s, y)}{s} \mathrm{~d} s+O\left(x^{\beta} Z(\beta, y) \mathrm{e}^{-c_{1}(\log u)^{4 / 3}}\right) .
$$


The last integral is classically evaluated by expanding the integrand around $s=\beta$. Let $T_{1}:=u^{-1 / 3} / \log y$. When $T_{1}<|\tau| \leqslant T_{0}$, we have

$$
Z(s, y) x^{s}=Z(\beta, y) x^{\beta} \mathrm{e}^{-\tau^{2} \sigma_{2} / 2+i \tau^{3} \sigma_{3} / 6+\sigma_{4} \tau^{4} / 24+O(1)} \ll Z(\beta, y) x^{\beta} \mathrm{e}^{-u^{1 / 3} / 5}
$$

by $(2 \cdot 20)$ and $(2 \cdot 21)$, since $\tau^{5} \varphi_{5}(\beta+i \tau, y) \ll T_{0}^{5} \sigma_{5} \ll 1$ for $|\tau| \leqslant T_{0}$. Therefore, we may replace $T_{0}$ by $T_{1}$ in $(3.5)$ without altering the error term.

We now evaluate the new main term.

Since $T_{1}^{j} \sigma_{j} \ll 1$ for $j=3,4$ and $\varphi_{4}(\beta+i \tau, y) \ll \sigma_{4}^{*}:=u(\log y)^{4}$ for $|\tau| \leqslant 1 / \log y$, we may write

$$
\begin{aligned}
\frac{1}{2 \pi i} \int_{\beta-i T_{1}}^{\beta+i T_{1}} \frac{x^{s} Z(s, y)}{s} \mathrm{~d} s & =\frac{x^{\beta} Z(\beta, y)}{2 \pi} \int_{-T_{1}}^{T_{1}} \mathrm{e}^{-\tau^{2} \sigma_{2} / 2+i \tau^{3} \sigma_{3} / 6+O\left(\tau^{4} \sigma_{4}^{*}\right)} \frac{\mathrm{d} \tau}{\beta+i \tau} \\
& =\frac{x^{\beta} Z(\beta, y)}{2 \pi}\left\{J_{2}+\frac{1}{6} i J_{3}+O(K)\right\}
\end{aligned}
$$

with

$$
\begin{aligned}
& J_{2}:=\int_{-T_{1}}^{T_{1}} \mathrm{e}^{-\tau^{2} \sigma_{2} / 2} \frac{\mathrm{d} \tau}{\beta+i \tau}, \quad J_{3}:=\sigma_{3} \int_{-T_{1}}^{T_{1}} \tau^{3} \mathrm{e}^{-\tau^{2} \sigma_{2} / 2} \frac{\mathrm{d} \tau}{\beta+i \tau}, \\
& K:=\int_{-T_{1}}^{T_{1}} \mathrm{e}^{-\tau^{2} \sigma_{2} / 2}\left\{\tau^{4} \sigma_{4}^{*}+\tau^{6} \sigma_{3}^{2}\right\} \frac{\mathrm{d} \tau}{|\beta|+|\tau|} .
\end{aligned}
$$

The required formula now follows from computations identical to those appearing in the end of the proof of proposition 2.13 of [2], so we do not repeat the details here.

This completes the proof of $(1 \cdot 11)$.

\subsection{Proof of Corollary 1.2}

We start with establishing (1.18). When $y>(\log x)^{3}$, we have, for $\gamma=\alpha$ or $\gamma=\beta$,

$$
\mathcal{H}_{y}(\gamma)=1+O\left(\frac{V_{y}(\gamma)}{\log y}\right)=1+O\left(\frac{u \log 2 u}{\sqrt{y} \log y}\right)
$$

by $(2 \cdot 6),(1 \cdot 14)$, and $(2 \cdot 9)$. Thus $(1 \cdot 18)$ holds in this case - and actually so do $(1 \cdot 21)$ and $(1 \cdot 22)$.

We may therefore assume $y \leqslant(\log x)^{3}$ henceforth.

By $(1 \cdot 11),(1 \cdot 14),(2 \cdot 10),(2 \cdot 4),(2 \cdot 21)$, and the definition of $\alpha$, we have for $2 \log x<$ $\psi(y) \ll(\log x)^{3}$,

$$
\Upsilon(x, y) \asymp \frac{x^{\beta} Z(\beta, y)}{1+\beta \sqrt{\sigma_{2}}} \gg \frac{\mathcal{H}_{y}(\beta) x^{\alpha} \zeta(\alpha, y)}{\alpha \sqrt{u} \log y} \asymp \mathcal{H}_{y}(\beta) \Psi(x, y)
$$

where the last estimate readily follows from the saddle-point asymptotic formula for $\Psi(x, y)$ - see [5], th. 1 -, viz.

$$
\Psi(x, y)=\frac{x^{\alpha} \zeta(\alpha, y)}{\alpha \sqrt{2 \pi g_{-}^{\prime}(\alpha, y)}}\left\{1+O\left(\frac{1}{u}+\frac{\log y}{y}\right)\right\} \quad(x \geqslant y \geqslant 2),
$$

where

$$
\zeta(s, y):=\prod_{p \leqslant y}\left(\frac{1}{1-p^{-s}}\right) \quad(\Re e s>0) .
$$

This completes the proof of the lower bound in (1.18). 
To prove the upper bound, we let $\varepsilon$ be a small positive constant and note that, if $\psi(y)>(2+\varepsilon) \log x$, we have $\alpha \asymp \beta$ by (1.13) and (2.10). We hence deduce from (1.11), $(1 \cdot 9),(2 \cdot 4)$ and $(2 \cdot 21)$ that

$$
\Upsilon(x, y) \asymp \frac{x^{\beta} Z(\beta, y)}{1+\beta \sqrt{\sigma_{2}}} \ll \frac{x^{\alpha} Z(\alpha, y)}{\alpha \sqrt{u} \log y} \asymp \mathcal{H}_{y}(\alpha) \Psi(x, y),
$$

where the first upper bound is an immediate consequence of the definition of $\beta$ and the latter follows again from $(3 \cdot 8)$.

When $\psi(y) \leqslant(2+\varepsilon) \log x$, the above argument is not sufficient since $\beta \log y$ may approach 0 while we always have $\alpha \gg 1 / \log y$. However, by Taylor's formula to the second order and the definition of $\beta$, we have

$$
x^{\beta} Z(\beta, y) \leqslant x^{\alpha} Z(\alpha, y) \mathrm{e}^{-v(\alpha-\beta)^{2}}
$$

where $v:=\frac{1}{2} \varphi_{2}(\gamma, y)$ for some $\left.\gamma \in\right] \beta, \alpha[$-it indeed follows from $(1 \cdot 14)$ and $(2 \cdot 10)$ that $\beta<\alpha$ if $\varepsilon$ is chosen sufficiently small. Now, we observe that, from lemma 13 of [5] and (1.13), we have $g_{+}^{\prime}(\alpha, y) \asymp u(\log y)^{2}$. Since $g_{+}^{\prime}(\sigma, y)$ is a non-increasing function of $\sigma$, we infer, taking $(2 \cdot 22)$ with $j=2$ and $(2 \cdot 23)$ into account, that $\varphi_{2}(\gamma, y) \asymp u(\log y)^{2}$. It remains to use the fact that $\alpha-\beta \asymp \alpha$ in the range under consideration to obtain, for some suitable absolute constant $c>0$,

$$
\Upsilon(x, y) \ll x^{\beta} Z(\beta, y) \ll x^{\alpha} Z(\alpha, y) \mathrm{e}^{-c u} \ll \mathcal{H}_{y}(\alpha) \Psi(x, y) \mathrm{e}^{-c u / 2} .
$$

This completes the proof of $(1 \cdot 18)$.

We next turn our attention to $(1 \cdot 21)$ and $(1 \cdot 22)$, which contain the sufficiency part of assertions $(1 \cdot 19)$ and $(1 \cdot 20)$ respectively.

In view of $(3 \cdot 6)$, we may assume that $(\log x)^{3 / 2} / \sqrt{\log _{2} x}<y \leqslant(\log x)^{3}$, and note that this is equivalent to $y \gg u^{3 / 2} \log 2 u$ and implies $\log y \asymp \log u \asymp \log _{2} x$. Since $\beta \sqrt{\sigma_{2}} \asymp \sqrt{u} \log y$ in the range under consideration, it follows from (1.9) that

$$
G\left(\beta \sqrt{\sigma_{2}}\right)=\frac{1+O(1 / u)}{\beta \sqrt{2 \pi \sigma_{2}}} .
$$

From $(2 \cdot 22)$, we see that, in this last expression, we can replace $\sigma_{2}$ by $g_{+}^{\prime}(\beta, y)$ at the cost of increasing the error to $\ll 1 / \sqrt{u}$. Furthermore, we observe that, in view of $(3 \cdot 13)$, we have

$$
\begin{aligned}
g_{-}^{\prime}(\beta, y)-g_{+}^{\prime}(\beta, y) & \ll \sum_{p \leqslant y} \frac{(\log p)^{2}}{p^{2 \beta}} \\
& \ll \frac{\left(y^{1-2 \beta}-1\right) \log y}{1-2 \beta} \asymp \frac{\left\{y+(\log x)^{2}\right\}(\log y)^{2}}{y(1+|\log r|)} \\
& \ll g_{-}^{\prime}(\beta, y) \frac{y+(\log x)^{2}}{u y(1+|\log r|)} \ll \frac{g_{-}^{\prime}(\beta, y)}{\sqrt{u}},
\end{aligned}
$$

where we used $(2 \cdot 19),(2 \cdot 7)$ and $(2 \cdot 17)$. It follows that

$$
\frac{x^{\beta} Z(\beta, y)}{\beta \sqrt{2 \pi \sigma_{2}}}=\frac{\mathcal{H}_{y}(\beta) x^{\beta} \zeta(\beta, y)}{\beta \sqrt{2 \pi g_{-}^{\prime}(\beta, y)}}\left\{1+O\left(\frac{1}{\sqrt{u}}\right)\right\} .
$$

Replacing $\beta$ by $\alpha$ in all terms except $\mathcal{H}_{y}(\beta)$ on the right-hand side yields an extra factor

$$
F:=\exp \left\{O\left(|\beta-\alpha| D+(\beta-\alpha)^{2} u(\log y)^{2}\right)\right\}
$$


with $D:=1 / \beta+g_{-}^{\prime \prime}(\beta, y) / g_{-}^{\prime}(\beta, y) \ll \log y$. In view of $(2 \cdot 11)$, we obtain, writing $L:=1+|\log r|$, that

$$
F-1 \ll \frac{\log 2 u}{\sqrt{y} L}+\frac{u(\log u)^{2}}{y L}+\frac{u^{3}(\log u)^{4}}{y^{2} L^{2}} \ll \frac{1}{\sqrt{u}}+\frac{u^{3}(\log u)^{2}}{y^{2}} \asymp E .
$$

By $(3 \cdot 8)$, we hence infer that $(1 \cdot 22)$ holds.

To prove $(1 \cdot 21)$, we assume $y \leqslant(\log x)^{3}$ and note that, for some $\vartheta \in[0,1]$ and $\gamma=\beta+\vartheta(\alpha-\beta)$, we have

$$
\mathcal{H}_{y}(\alpha)=\mathcal{H}_{y}(\beta) \mathrm{e}^{(\alpha-\beta) V_{y}(\gamma)} .
$$

By $(2 \cdot 11)$ and $(2 \cdot 18)$, which is equally valid for $\gamma$, we obtain, still using the notation $L:=1+|\log r|$,

$$
\log \left(\frac{\mathcal{H}_{y}(\alpha)}{\mathcal{H}_{y}(\beta)}\right) \asymp \frac{u(\log y)^{2}}{y L^{2}}+\frac{u^{3}(\log y)^{4}}{y^{2} L^{2}} \ll \frac{1}{\sqrt{u}}+\frac{(\log x)^{3}}{y^{2} \log _{2} x} \asymp E .
$$

Thus, we have established $(1 \cdot 21)$.

It remains to prove that the asymptotic formulae in (1.19) and (1.20) do not hold when $y \ll(\log x)^{3 / 2} / \sqrt{\log _{2} x}$. From $(3 \cdot 7)$, we have in this range

$$
\Upsilon(x, y) \gg \mathcal{H}_{y}(\beta) \Psi(x, y) \mathrm{e}^{(\alpha-\beta)^{2} g_{-}^{\prime}(\gamma, y) / 2}
$$

for some $\gamma:=\alpha+\vartheta(\beta-\alpha)$ with $0 \leqslant \vartheta \leqslant 1$. However, estimates $(2 \cdot 17)$ and (2.11) yield in this case

$$
(\alpha-\beta)^{2} g_{-}^{\prime}(\gamma, y) \asymp \frac{(\log x)^{3}}{y^{2} \log _{2} x} \gg 1 .
$$

Hence the asymptotic formula in $(1 \cdot 20)$ cannot hold.

We have already seen in $(3 \cdot 10)$ that $(1 \cdot 19)$ fails when $\psi(y) \leqslant(2+\varepsilon)$ with $\varepsilon$ sufficiently small. In the complementary range, the asymptotic formula (1.11) yields

$$
\Upsilon(x, y) \asymp \frac{x^{\alpha} Z(\alpha, y)}{\alpha \sqrt{u} \log y} \mathrm{e}^{-(\alpha-\beta)^{2} \varphi_{2}(\gamma, y) / 2} \asymp \mathcal{H}_{y}(\alpha) \Psi(x, y) \mathrm{e}^{-(\alpha-\beta)^{2} \varphi_{2}(\gamma, y) / 2}
$$

for some $\gamma$ between $\alpha$ and $\beta$. But $(2 \cdot 17)$ and the last estimate of $(2 \cdot 18)$, which also holds for $\gamma$ in place of $\beta$, yield $\varphi_{2}(\gamma, y) \asymp u(\log y)^{2}$. Hence we get as before

$$
(\alpha-\beta)^{2} \varphi_{2}(\gamma, y) \asymp \frac{(\log x)^{3}}{y^{2} \log _{2} x} \gg 1 .
$$

This completes the proof of assertion (1.19).

\section{3. Proof of Corollary 1.3}

We observe at the outset that the asymptotic formulae $(1.24)$ and (1.25) readily follow, respectively, from $(1 \cdot 10)$ when $y>(\log x)^{2+\varepsilon}$, and from $(1 \cdot 18)$ when

$$
\log x \ll y \leqslant(\log x)^{2} / L_{\varepsilon}(\log x)
$$

with, say $0<\varepsilon<\frac{1}{10}$. Indeed, for large $x$, we then have $\alpha \leqslant \frac{1}{2}-1 /\left(\log _{2} x\right)^{2 / 5+2 \varepsilon}$, and so

$$
\mathcal{H}_{y}(\alpha) \ll \exp \left\{-\sum_{\sqrt{y} \leqslant p \leqslant y} 1 / p^{2 \alpha}\right\} \ll \frac{1}{L_{3 \varepsilon}(\log x)}=o(1) .
$$

Moreover, in view of (1.23), it is clear that, in the stated range of validity, (1.26) implies $(1 \cdot 24)$ and $(1 \cdot 25)$ since it may be easily checked that $h$ and $\log \left\{y /(\log x)^{2}\right\}$ tend simultaneously to $\pm \infty$.

Thus it only remains to establish (1·26). 
We note that the first formula in (1.26) readily follows from (1.22) since, in the considered range, we have $E \ll 1 / \sqrt{u}$.

We next need an estimate for $\mathcal{H}_{y}(\beta)$. We plainly have $y \leqslant(\log x)^{3}$. We may hence deduce from $(2 \cdot 10)$ that, for a suitable constant $c>0$, we have

$$
\beta \geqslant \frac{1}{2}-\frac{c}{(\log y)^{2 / 5+\varepsilon}}
$$

It follows from $(3 \cdot 13)$ that

$$
\prod_{p \leqslant \sqrt{y}}\left(1-p^{-\left(\nu_{p}+1\right) \beta}\right)=\exp \left\{O\left(\frac{y^{(1-3 \beta) / 2}-y^{(1-3 \beta) / 3}}{(1-3 \beta) \log y}\right)\right\}=1+O\left(\frac{u \log u}{y^{2 / 3} \log y}\right) .
$$

Since $\log u \asymp \log y$, we get

$$
\mathcal{H}_{y}(\beta)=\left\{1+O\left(\frac{u}{y^{2 / 3}}\right)\right\} \prod_{\sqrt{y}<p \leqslant y}\left(1-p^{-2 \beta}\right) .
$$

We now apply (2.9) with $\varepsilon / 2$ instead of $\varepsilon$ and consequently replace $\beta$ by $1-\xi(u) / \log y=$ $\frac{1}{2}+h / \log y$ in the latter product. This involves an extra factor

$$
\exp \left\{O\left(\sum_{\sqrt{y}<p \leqslant y} \frac{\log p}{p^{2 \beta} L_{\varepsilon / 2}(y)}\right)\right\}=1+O\left(\frac{1}{L_{\varepsilon}(y)}\right) .
$$

Now, we have by lemma III.5.16 of [11]

$$
\prod_{\sqrt{y}<p \leqslant y}\left(1-p^{-1-2 h / \log y}\right)=\frac{\widehat{\varrho}(h)}{2 \widehat{\varrho}(2 h)}\left\{1+O\left(\frac{1}{L_{\varepsilon}(y)}\right)\right\} \quad(y \geqslant 2) .
$$

Gathering our estimates so far, we obtain

$$
\mathcal{H}_{y}(\beta)=\frac{\widehat{\varrho}(h)}{2 \widehat{\varrho}(2 h)}\left\{1+O\left(\frac{1}{L_{\varepsilon}(y)}\right)\right\} \quad(y \geqslant 2) .
$$

Inserting this into (1.22), we obtain the second formula in (1.26), as required.

Acknowledgement. The author takes pleasure in expressing here his gratitude to the anonymous referee, whose remarks and suggestions significantly helped clarifying this article.

\section{References}

[1] A.I. Bonciocat \& N.C. Bonciocat, On the irreducibility of polynomials with leading coefficient divisible by a large prime power, Amer. Math. Monthly 116, no 8 (2009), 743-745.

[2] R. de la Bretèche \& G. Tenenbaum, Sur les lois locales de la répartition du $k$-ième diviseur d'un entier, Proc. London Math. Soc. (3) 84 (2002), 289-323.

[3] R. de la Bretèche \& G. Tenenbaum, Propriétés statistiques des entiers friables, Ramanujan J. 9 (2005), 139-202.

[4] J.-M. De Koninck, F. Luca \& L. Szalay, A Schinzel hypothesis H type of result for economical numbers, Ann. Sci. Math. Québec 29, no 1 (2005), 35-39.

[5] A. Hildebrand \& G. Tenenbaum, On integers free of large prime factors, Trans. Amer. Math. Soc. 296 (1986), 265-290.

[6] A. Hildebrand \& G. Tenenbaum, On a class of difference differential equations arising in number theory, J. d'Analyse 61 (1993), 145-179. 
[7] V. V. Petrov, Sums of independent random variables, Springer Verlag, Berlin, Heidelberg, New York, 1975.

[8] O. Robert \& G. Tenenbaum, Sur la répartition du noyau d'un entier, Indag. Math. 24 (2013), 802-914.

[9] J.W. Sander, Integral circulant Ramanujan graphs via multiplicativity, preprint, 2014.

[10] G. Tenenbaum, Sur un problème extrémal en Arithmétique, Ann. Inst. Fourier (Grenoble) 37, 2 (1987), 1-18; Corrigendum, ibid. 50, 1 (2000), 311-312.

[11] G. Tenenbaum, Introduction à la théorie analytique et probabiliste des nombres, troisième édition, coll. Échelles, Belin, 2008, 592 pp.

Gérald Tenenbaum

Institut Élie Cartan

Université de Lorraine

BP 70239

54506 Vandouvre Cedex

France

gerald.tenenbaum@univ-lorraine.fr 